\title{
Specific effects of dietary methylmercury and inorganic mercury in zebrafish (Danio rerio) determined by genetic, histological and metallothionein responses.
}

Sophie Gentès †, Régine Maury-Brachet †, Caiyan Feng \|, Zoyne Pedrero \|, Emmanuel Tessier $\|$, Alexia Legeay $\dagger$, Nathalie Mesmer-Dudons $\uparrow$, Magalie Baudrimont $\uparrow$, Laurence Maurice $¥ \S$, David Amouroux $\|$, Patrice Gonzalez * $\$$.

† Université de Bordeaux, EPOC, UMR CNRS 5805, Place du Dr B. Peyneau F-33120 Arcachon, France.

$\$$ CNRS, EPOC, UMR 5805, F-33120 Arcachon, France.

\| Laboratoire de Chimie Analytique, Bio-Inorganique et Environnement, Institut des Sciences Analytiques et de Physico-Chimie pour l'Environnement et les Matériaux (IPREM), CNRS-UPPA-UMR-5254, Hélioparc, 2 Avenue du Président Pierre Angot, F-64053 Pau, France.

$\S$ Observatoire Midi-Pyrénées, Laboratoire de Geosciences Environnement Toulouse, Université Paul Sabatier Toulouse III, 14 avenue Edouard Belin, 31400 Toulouse, France.

‡ GET, IRD, F-31400 Toulouse, France.

*Corresponding author: Patrice Gonzalez. Phone: + (33) 05562239 21; Fax : +(33) 05565493 83; email: p.gonzalez@epoc.u-bordeaux1.fr

\section{SUPPORTING INFORMATION}

5 tables; 2 figures; 8 pages 
Experimental design.

Fish were maintained in the laboratory for 20 days prior to experimentation and fed with the same food as the fish breeder in order to minimize stress due to the change of environment, which may affect the results. Adult zebrafish were randomly placed in 10 tanks (66 fish for one tank: control at 0 day; 86 fish per tank for the others) containing $70 \mathrm{~L}$ of chlorine-free water, continuously oxygenated.

For each D. rerio, skeletal muscle, brain and liver were independently harvested. Brain and liver were collected in their entirety. The two skeletal muscle fillets were taken from the dorsal region between the head and the tail.

For gene expression analysis and metallothionein (MT) analysis, five replicates were constituted by pooling organs from four fish and then divided for the different analyses (chemical analyses). For Hg dosages, three replicates were carried out by pooling organs from three other fish.

Table 1S. Theoretical quantity of mercury absorbed per fish during experiment.

\begin{tabular}{|c|c|c|c|c|}
\hline Condition & $\begin{array}{l}\text { theoretical } \\
\text { average } \\
\mathrm{Hg} / \text { fish/day } \\
\text { (ng) }\end{array}$ & $\begin{array}{l}\text { theoretical } \\
\mathrm{Hg} / \text { fish at } \\
7 \text { days (ng) }\end{array}$ & $\begin{array}{c}\text { theoretical } \\
\mathrm{Hg} / \text { fish at } \\
25 \text { days } \\
\text { (ng) }\end{array}$ & $\begin{array}{c}\text { theoretical } \\
\mathrm{Hg} / \text { fish at } \\
62 \text { days } \\
\text { (ng) }\end{array}$ \\
\hline Control & 1 & 4 & 18 & 50 \\
\hline $\mathrm{iHg}$ & 176 & 894 & 3916 & 10883 \\
\hline $\mathrm{MeHg}$ & 171 & 869 & 3805 & 10575 \\
\hline
\end{tabular}


Figure 1S. Average total mercury (THg) concentrations in the skeletal muscle, liver and brain of Danio rerio after 7, 25, and 62 days of dietary exposure to $\mathrm{MeHg}$ and $\mathrm{iHg}$. Analysis were determined by flameless atomic absorption spectrometry (AMA 254, Prague, Czech Republic). A: the three conditions (low-MeHg, MeHg, $\mathrm{iHg}$ ). B: focus on the low-MeHg and $\mathrm{iHg}$ conditions. Error bars represent standard deviations, $\mathrm{n}=3$ to 4 .

Letters indicate statistical differences $(\mathrm{p}<0.05)$.
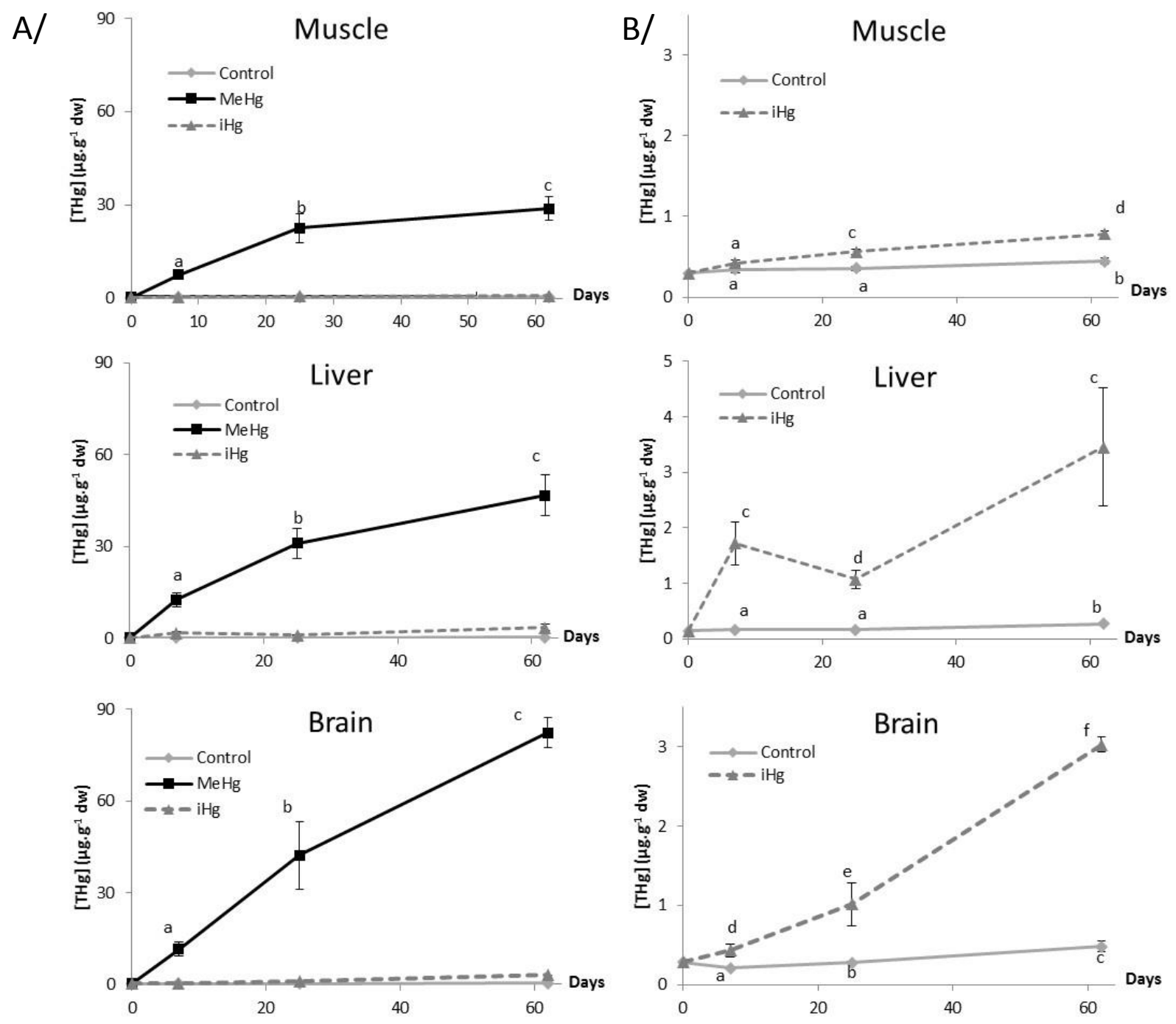
Table 2S. Function, accession numbers and specific primer matching with Danio rerio genes used for quantitative RT-PC

\begin{tabular}{|c|c|c|c|c|}
\hline Gene symbol & Gene name & Function & Accession number & Primer Sequence (5'-3') \\
\hline bax & bcl2-associated $X$ protein & \multirow{4}{*}{ apoptosis } & \multirow{2}{*}{ AF231015 } & F:GGCTATTTCAACCAGGGTTCC \\
\hline & & & & R: TGCGAATCACCAATGCTGT \\
\hline 053 & tumor protein p53 & & \multirow{2}{*}{ AF365873 } & F: GGGCAATCAGCGAGCAAA \\
\hline (20 & (1) & & & R: ACTGACCTTCCTGAGTCTCCA \\
\hline $\operatorname{rad51}$ & RAD51 homolog & DNA repair & BC062849 & $\begin{array}{l}\text { F: TGCTGCGTCTCGCTGA } \\
\text { R: GCCTCGGCCTCTGGTAA }\end{array}$ \\
\hline tap & $\begin{array}{l}\text { transporter 1, ATP-binding cassette, sub- } \\
\text { family B (MDR/TAP) }\end{array}$ & \multirow{3}{*}{$\begin{array}{c}\text { cellular } \\
\text { detoxification }\end{array}$} & AL672164 & $\begin{array}{l}\text { F: GCAAGATAGCGATGGTGGG } \\
\text { R: CGTTGGCTTTGCTTGCAG }\end{array}$ \\
\hline$m+2$ & metallothionein-2 & & AY305851 & $\begin{array}{l}\text { F: TGCGAATGCGCCAAGAC } \\
\text { R: GCCCTTACACACGCAGC }\end{array}$ \\
\hline hsf1 & heat shock trancription factor 1 & & NM_131600.1 & $\begin{array}{l}\text { F: GGACACGCCCCTTTCG } \\
\text { R: CTGAATGAGGCCGGAGGT }\end{array}$ \\
\hline gpx4a & Glutathione peroxydase $4 a$ & \multirow{3}{*}{$\begin{array}{l}\text { oxidative } \\
\text { stress } \\
\text { response }\end{array}$} & NM_001007282.1 & $\begin{array}{l}\text { F: CCGTGAAGGCCAGGTTG } \\
\text { R: GCCCTCAGGAGGAGGTG }\end{array}$ \\
\hline sod & cytoplasmic superoxide dismutase & & BC055516 & $\begin{array}{l}\text { F: TGAGACACGTCGGAGACC } \\
\text { R:TGCCGATCACTCCACAGG }\end{array}$ \\
\hline sod $m t$ & mitochondrial superoxide dismutase & & CB923500 & $\begin{array}{l}\text { F: TTCAGGGCTCAGGCTGG } \\
\text { R: ATGGCTTTAACATAGTCCGGT }\end{array}$ \\
\hline vdac2 & Voltage-dependent anion channel 2 & \multirow{3}{*}{$\begin{array}{l}\text { mitochondrial } \\
\text { metabolism }\end{array}$} & BC164640.1 & $\begin{array}{l}\text { F: ACCTGGGATGTGATGTTGAC } \\
\text { R: ACCACCAAACTCTGAGCC }\end{array}$ \\
\hline $\operatorname{cox} 1$ & cytochrome $\mathrm{c}$ oxidase 1 & & NC_002333 & $\begin{array}{l}\text { F: GGAATACCACGACGGTACTCT } \\
\text { R: AGGGCAGCCGTGTAAT } \\
\text { F: ACTTTCTACCCCAAATATAC }\end{array}$ \\
\hline $12 S$ & Ribosomal RNA small subunit & & NC_002333.2 & R: CACATCCTTCCAC \\
\hline laptm4a & $\begin{array}{l}\text { Lysosomal associated protein } \\
\text { transmembrane } 4 \text { alpha }\end{array}$ & $\begin{array}{l}\text { proteins } \\
\text { transporter }\end{array}$ & NM_214685.2 & $\begin{array}{l}\text { F: AGATTGCTGTCTATCCTGCTT } \\
\text { R: GTGGACGCTGGGAGTCTA }\end{array}$ \\
\hline gfap & glial fibrillary acidic protein & & NM_131373.2 & $\begin{array}{l}\text { F: GGAGTACCAGGACCTGCT } \\
\text { R: CTCCGTCACGGGTCTCAA }\end{array}$ \\
\hline$s c / 1 A 3 b$ & excitatory amino acid transporter & $\begin{array}{c}\text { nervous } \\
\text { transmission }\end{array}$ & HM138694.1 & $\begin{array}{l}\text { F: CTTGACGAACGCTACGCAG } \\
\text { R: AGTCGCATGATGGCCT }\end{array}$ \\
\hline ache & acetylcholinesterase & & NM_131846.1 & $\begin{array}{l}\text { F: ACTCGCATGGTGCTGT } \\
\text { R: CCAGTGCGGGCGAAAT }\end{array}$ \\
\hline$h s d 3 b 1$ & $\begin{array}{l}\text { Hydroxy-delta-5-steroid dehydrogenase, } 3 \\
\text { beta- and steroid delta-isomerase } 1\end{array}$ & lipid & NM_212797.1 & $\begin{array}{l}\text { F: ATGCGATCCGCAGTGG } \\
\text { R: AGCATGGGTTAAGTCACAATAGC }\end{array}$ \\
\hline apoeb & apolipoprotein Eb & metabolism & NM_131098.1 & $\begin{array}{l}\text { F: ACATGACCGACGCTAAGG } \\
\text { R: CGCGGGACTGCATCTC }\end{array}$ \\
\hline ef1a1 & $\begin{array}{c}\text { eukaryotic translation elongation factor } 1 \\
\text { alpha } 1\end{array}$ & & AY422992.1 & $\begin{array}{l}\text { F:GGGAAAGGAAAAGACCCACA } \\
\text { R:CGCTCGGCCTTCAGTT }\end{array}$ \\
\hline$r p / 13 a$ & ribosomal protein $\mathrm{L} 13 \mathrm{a}$ & $\begin{array}{l}\text { reference } \\
\text { gene }\end{array}$ & NM_212784.1 & $\begin{array}{l}\text { F: ACCCTTCCCGTGGATCA } \\
\text { R: GTGGGTTTCAGACGCACA }\end{array}$ \\
\hline$B$-actin & bactin1 & & NM 131031 & $\begin{array}{l}\text { F: AAGTGCGACGTGGACA } \\
\text { R: GTTTAGGTTGGTCGTTCGTTTGA }\end{array}$ \\
\hline
\end{tabular}


Table 3S. Differential gene expression as compared to $\beta$-actin, rpll3a and EEF1A1 genes in muscle from Danio rerio after 7, 25 and 62 days of dietary exposure to $\mathrm{MeHg}$ and $\mathrm{iHg}$. Low-MeHg: no $\mathrm{Hg}$ added; $\mathrm{MeHg}$ and $\mathrm{iHg}$ : $171 \mathrm{ng}$. fish ${ }^{-1}$. day ${ }^{-1}$. Value \pm standard deviation, $\mathrm{n}=3$ to 5 .

\begin{tabular}{|c|c|c|c|c|c|c|c|c|c|c|}
\hline \multirow[b]{2}{*}{ gene } & \multirow[b]{2}{*}{ function } & \multicolumn{3}{|c|}{ Low- MeHg } & \multicolumn{3}{|c|}{ MeHg } & \multicolumn{3}{|c|}{$\mathrm{iHg}$} \\
\hline & & 7 & 25 & 62 & 7 & 25 & 62 & 7 & 25 & 62 \\
\hline$b a x$ & anontocic & $0.0012 \pm 0.0002$ & $0.0020 \pm 0.0005$ & $0.0019 \pm 0.0006$ & $0.0007 \pm 0.0004$ & $0.0012 \pm 0.0007$ & $0.0024 \pm 0.0008$ & $0.0013 \pm 0.0012$ & $0.0016 \pm 0.0002$ & $0.0020 \pm 0.0007$ \\
\hline$p 53$ & apoptosis & $0.013 \pm 0.002$ & $0.029 \pm 0.008$ & $0.018 \pm 0.003$ & $0.013 \pm 0.008$ & $0.021 \pm 0.011$ & $0.016 \pm 0.004$ & $0.013 \pm 0.007$ & $0.027 \pm 0.008$ & $0.020 \pm 0.004$ \\
\hline rad51 & DNA repair & $0.004 \pm 0.003$ & $0.019 \pm 0.016$ & $0.004 \pm 0.005$ & $0.003 \pm 0.005$ & $0.016 \pm 0.014$ & $0.005 \pm 0.004$ & $0.003 \pm 0.002$ & $0.016 \pm 0.009$ & $0.002 \pm 0.001$ \\
\hline tap & cellular detoxification & $0.011 \pm 0.005$ & $0.023 \pm 0.010$ & $0.033 \pm 0.013$ & $0.018 \pm 0.009$ & $0.012 \pm 0.006$ & $0.021 \pm 0.012$ & $0.012 \pm 0.009$ & $0.009 \pm 0.005$ & $0.022 \pm 0.011$ \\
\hline$m+2$ & and response to & $0.033 \pm 0.035$ & $0.082 \pm 0.050$ & $0.162 \pm 0.135$ & $0.014 \pm 0.017$ & $0.092 \pm 0.030$ & $0.125 \pm 0.094$ & $0.071 \pm 0.069$ & $0.556 \pm 0.353$ & $0.105 \pm 0.043$ \\
\hline$h s f 1$ & oxidative stress & $0.006 \pm 0.002$ & $0.007 \pm 0.002$ & $0.010 \pm 0.002$ & $0.004 \pm 0.002$ & $0.006 \pm 0.005$ & $0.011 \pm 0.005$ & $0.006 \pm 0.002$ & $0.005 \pm 0.002$ & $0.019 \pm 0.007$ \\
\hline$g p \times 4 a$ & & $0.064 \pm 0.021$ & $0.14 \pm 0.09$ & $0.19 \pm 0.16$ & $0.08 \pm 0.04$ & $0.11 \pm 0.07$ & $0.09 \pm 0.07$ & $0.16 \pm 0.07$ & $0.08 \pm 0.03$ & $0.11 \pm 0.02$ \\
\hline sod & oxidative stress & $0.093 \pm 0.03$ & $0.223 \pm 0.032$ & $0.009 \pm 0.005$ & $0.003 \pm 0.001$ & $0.185 \pm 0.018$ & $0.049 \pm 0.063$ & $0.007 \pm 0.007$ & $0.134 \pm 0.055$ & $0.164 \pm 0.038$ \\
\hline sodmt & & $0.57 \pm 0.19$ & $0.93 \pm 0.50$ & $0.74 \pm 0.23$ & $0.69 \pm 0.26$ & $1.07 \pm 0.44$ & $0.57 \pm 0.19$ & $0.99 \pm 0.52$ & $0.76 \pm 0.24$ & $1.08 \pm 0.38$ \\
\hline vdac2 & & $0.52 \pm 0.25$ & $0.33 \pm 0.21$ & $0.69 \pm 0.24$ & $0.59 \pm 0.43$ & $0.32 \pm 0.17$ & $1.12 \pm 0.81$ & $0.56 \pm 0.18$ & $0.24 \pm 0.06$ & $2.09 \pm 0.86$ \\
\hline $\operatorname{coxl}$ & $\begin{array}{l}\text { mitochonarial } \\
\text { metabolism }\end{array}$ & $150.7 \pm 54$ & $264.4 \pm 173.9$ & $183.4 \pm 55.8$ & $128.4 \pm 59.6$ & $401.8 \pm 232.1$ & $134.1 \pm 80.7$ & $206.1 \pm 100.5$ & $211.2 \pm 82.8$ & $335.8 \pm 218.4$ \\
\hline $12 \mathrm{~s}$ & & $0.006 \pm 0.002$ & $0.003 \pm 0.002$ & $0.005 \pm 0.002$ & $0.004 \pm 0.003$ & $0.008 \pm 0.004$ & $0.007 \pm 0.008$ & $0.01 \pm 0.01$ & $0.004 \pm 0.002$ & $0.011 \pm 0.005$ \\
\hline laptm4 & protein transporter & $0.0015 \pm 0.0006$ & $0.0017 \pm 0.0016$ & $0.003 \pm 0.001$ & $0.0029 \pm 0.0034$ & $0.0017 \pm 0.0007$ & $0.0038 \pm 0.0032$ & $0.0023 \pm 0.0012$ & $0.0016 \pm 0.0010$ & $0.0106 \pm 0.0086$ \\
\hline gfap & & $0.001 \pm 0$ & $0.013 \pm 0.014$ & $0.012 \pm 0.013$ & $0.0004 \pm 0.0003$ & $0.0003 \pm 0.0002$ & $0.002 \pm 0.001$ & $0.001 \pm 0.001$ & $0.0006 \pm 0.0004$ & $0.018 \pm 0.019$ \\
\hline slc1a & nervous transmission & $0.0025 \pm 0.0014$ & $0.0035 \pm 0.0025$ & $0.0013 \pm 0.0005$ & $0.0018 \pm 0.0022$ & $0.0021 \pm 0.0015$ & $0.0013 \pm 0.0008$ & $0.0018 \pm 0.0015$ & $0.0026 \pm 0.0013$ & $0.0029 \pm 0.0012$ \\
\hline ache & & $0.11 \pm 0.03$ & $0.12 \pm 0.08$ & $0.24 \pm 0.05$ & $0.12 \pm 0.06$ & $0.07 \pm 0.03$ & $0.17 \pm 0.07$ & $0.020 \pm 0.002$ & $0.07 \pm 0.04$ & $0.26 \pm 0.08$ \\
\hline hsd3 & lipid metabolism & $5.3 \cdot 10^{-5} \pm 5 \cdot 4 \cdot 10^{-5}$ & $0,0003 \pm 0,0005$ & $3.3 .10^{-5} \pm 4.5 .10^{-5}$ & $4.5 \cdot 10^{-5} \pm 7.5 .10^{-5}$ & $0,0002 \pm 0,0002$ & $0,0001 \pm 0,0002$ & $6.2 \cdot 10^{-5} \pm 3.7 \cdot 10^{-5}$ & $0,0004 \pm 0,0002$ & $1.4 \cdot 10^{-5} \pm 1 \cdot 2 \cdot 10^{-5}$ \\
\hline apoeb & & $0.069 \pm 0.044$ & $0.118 \pm 0.074$ & $0.153 \pm 0.048$ & $0.069 \pm 0.044$ & $0.108 \pm 0.085$ & $0.075 \pm 0.032$ & $0.051 \pm 0.006$ & $0.065 \pm 0.026$ & $0.142 \pm 0.087$ \\
\hline
\end{tabular}


Table 4S. Differential gene expression as compared to $\beta$-actin, rpl13a and EEF1A1 genes in liver from Danio rerio after 7, 25 and 62 days of dietary exposure to $\mathrm{MeHg}$ and $\mathrm{iHg}$. Low-MeHg: no $\mathrm{Hg}$ added; $\mathrm{MeHg}$ and $\mathrm{iHg}: 171 \mathrm{ng}$. fish ${ }^{-1}$. day ${ }^{-1}$. Value \pm standard deviation, $\mathrm{n}=3$ to 5 .

\begin{tabular}{|c|c|c|c|c|c|c|c|c|c|c|}
\hline \multirow[b]{2}{*}{ gene } & \multirow[b]{2}{*}{ function } & \multicolumn{3}{|c|}{ Low- MeHg } & \multicolumn{3}{|c|}{ MeHg } & \multicolumn{3}{|c|}{$\mathrm{iHg}$} \\
\hline & & 7 & 25 & 62 & 7 & 25 & 62 & 7 & 25 & 62 \\
\hline$b a x$ & anontocic & $0.001 \pm 0.001$ & $0.008 \pm 0.011$ & $0.0012 \pm 0.0004$ & $0.0005 \pm 0.0002$ & - & $0.001 \pm 0.001$ & $0.008 \pm 0.017$ & $0.004 \pm 0.006$ & $0.002 \pm 0.001$ \\
\hline p53 & dpoptosis & $0.011 \pm 0.009$ & $0.007 \pm 0.004$ & $0.012 \pm 0.011$ & $0.007 \pm 0.005$ & $0.004 \pm 0.004$ & $0.006 \pm 0.003$ & $0.012 \pm 0.007$ & $0.006 \pm 0.001$ & $0.013 \pm 0.008$ \\
\hline $\operatorname{rad51}$ & DNA repair & $0.005 \pm 0.002$ & $0.003 \pm 0.003$ & $0.002 \pm 0.003$ & $0.002 \pm 0.001$ & $0.001 \pm 0.001$ & $0.002 \pm 0.001$ & $0.002 \pm 0.001$ & $0.004 \pm 0.001$ & $0.003 \pm 0.002$ \\
\hline tap & cellular detoxification & $0.021 \pm 0.022$ & $0.047 \pm 0.052$ & $0.009 \pm 0.004$ & $0.014 \pm 0.008$ & $0.019 \pm 0.011$ & $0.008 \pm 0.003$ & $0.016 \pm 0.012$ & $0.012 \pm 0.007$ & $0.022 \pm 0.013$ \\
\hline$m+2$ & and response to & $0.12 \pm 0.12$ & $0.23 \pm 0.08$ & $0.2 \pm 0.17$ & $0.02 \pm 0.01$ & $0.21 \pm 0.20$ & $0.08 \pm 0.11$ & $0.04 \pm 0.01$ & $0.28 \pm 0.23$ & $0.58 \pm 0.65$ \\
\hline hsf1 & oxidative stress & $0.002 \pm 0.002$ & $0.0006 \pm 0.0002$ & $0.001 \pm 0.001$ & $0.0003 \pm 0.0004$ & $0.0003 \pm 0.0002$ & $0.001 \pm 0.001$ & $0.001 \pm 0.002$ & $0.0005 \pm 0.0001$ & $0.003 \pm 0.002$ \\
\hline$g p \times 4 a$ & & $6.8 \pm 6.3$ & $7.0 \pm 4.3$ & $16.2 \pm 3.8$ & $6.1 \pm 3.7$ & $8.1 \pm 2.8$ & $16.3 \pm 12.0$ & $5.3 \pm 2.2$ & $5.9 \pm 2.0$ & $15.8 \pm 19.0$ \\
\hline sod & oxidative stress & $0.28 \pm 0.12$ & $0.40 \pm 0.17$ & $0.12 \pm 0.05$ & $0.02 \pm 0.01$ & $0.27 \pm 0.11$ & $0.34 \pm 0.36$ & $0.05 \pm 0.04$ & $0.56 \pm 0.22$ & $0.65 \pm 0.30$ \\
\hline sodmt & & $0.14 \pm 0.05$ & $0.33 \pm 0.10$ & $0.42 \pm 0.19$ & $0.21 \pm 0.10$ & $0.34 \pm 0.08$ & $0.61 \pm 0.53$ & $0.23 \pm 0.06$ & $0.55 \pm 0.11$ & $0.46 \pm 0.14$ \\
\hline vdac2 & & $0.05 \pm 0.05$ & $0.02 \pm 0.01$ & $0.03 \pm 0.02$ & $0.03 \pm 0.02$ & $0.008 \pm 0.005$ & $0.02 \pm 0.01$ & $0.03 \pm 0.03$ & $0.01 \pm 0.01$ & $0.04 \pm 0.03$ \\
\hline $\operatorname{coxl}$ & mitochondrial & $34.7 \pm 25.7$ & $305.2 \pm 394.5$ & $117.4 \pm 73.3$ & $42.0 \pm 27.9$ & $437.3 \pm 492.4$ & $292.4 \pm 366.2$ & $74.6 \pm 81.3$ & $341.7 \pm 213.2$ & $108.8 \pm 89.0$ \\
\hline $12 \mathrm{~s}$ & & $0.002 \pm 0.001$ & $0.006 \pm 0.003$ & $0.008 \pm 0.004$ & $0.001 \pm 0.001$ & $0.017 \pm 0.012$ & $0.004 \pm 0.003$ & $0.004 \pm 0.005$ & $0.007 \pm 0.006$ & $0.005 \pm 0.003$ \\
\hline laptm4 & protein transporter & $0.002 \pm 0.002$ & $0.0009 \pm 0.0005$ & $0.002 \pm 0.002$ & $0.0004 \pm 0.0001$ & $0.001 \pm 0.001$ & $0.001 \pm 0.001$ & $0.003 \pm 0.004$ & $0.001 \pm 0.0004$ & $0.005 \pm 0.003$ \\
\hline gfap & & $0.0003 \pm 0.0001$ & $2.5 .10^{-5} \pm 2.7 .10^{-6}$ & $2.9 .10^{-5} \pm 4.5 .10^{-5}$ & $1.2 \cdot 10^{-5} \pm 1.3 .10^{-5}$ & $2.6 .10^{-5} \pm 8.2 .10^{-6}$ & $1.7 \cdot 10^{-5} \pm 1.1 .10^{-5}$ & $3.6 .10^{-5} \pm 3.1 .10^{-5}$ & $2.3 \cdot 10^{-5} \pm 2.0 .10^{-5}$ & $5.8 .10^{-5} \pm 3.7 .10^{-5}$ \\
\hline$s / c 1 a$ & nervous transmission & $0.001 \pm 0.001$ & $0.007 \pm 0.004$ & $0.003 \pm 0.002$ & $0.001 \pm 0.001$ & $0.018 \pm 0.015$ & $0.005 \pm 0.006$ & $0.002 \pm 0.002$ & $0.018 \pm 0.015$ & $0.003 \pm 0.003$ \\
\hline ache & & $0.0019 \pm 0.002$ & $0.0012 \pm 0.0009$ & $0.0006 \pm 0.0006$ & $0.0008 \pm 0.0005$ & $0.0006 \pm 0.0005$ & $0.0011 \pm 0.0014$ & $0.0022 \pm 0.0015$ & $0.0006 \pm 0.0002$ & $0.0022 \pm 0.0013$ \\
\hline$h s d 3$ & linid metaholism & $9.8 \cdot 10^{-5} \pm 1.1 .10^{-4}$ & $0.0006 \pm 0.0005$ & $0.0002 \pm 0.0002$ & $3.6 .10^{-5} \pm 2.9 .10^{-5}$ & $0.001 \pm 0.001$ & $0.001 \pm 0.002$ & $0.0001 \pm 0.0001$ & $0.002 \pm 0.002$ & $0.0002 \pm 0.0003$ \\
\hline apoeb & ippia metadoonsm & $0.28 \pm 0.05$ & $0.38 \pm 0.08$ & $0.34 \pm 0.24$ & $0.44 \pm 0.23$ & $0.71 \pm 0.30$ & $0.88 \pm 0.55$ & $0.66 \pm 0.67$ & $0.35 \pm 0.11$ & $3.30 \pm 3.63$ \\
\hline
\end{tabular}


Table 5S. Differential gene expression as compared to $\beta$-actin, rpl13a and EEF1A1 genes in brain from Danio rerio after 7, 25, and 62 days of dietary exposure to $\mathrm{MeHg}$ and iHg. Low-MeHg: no $\mathrm{Hg}$ added; $\mathrm{MeHg}$ and $\mathrm{iHg}$ : $171 \mathrm{ng}$. fish ${ }^{-1}$. day ${ }^{-1}$.Value \pm standard deviation, $\mathrm{n}=3$ to 5 .

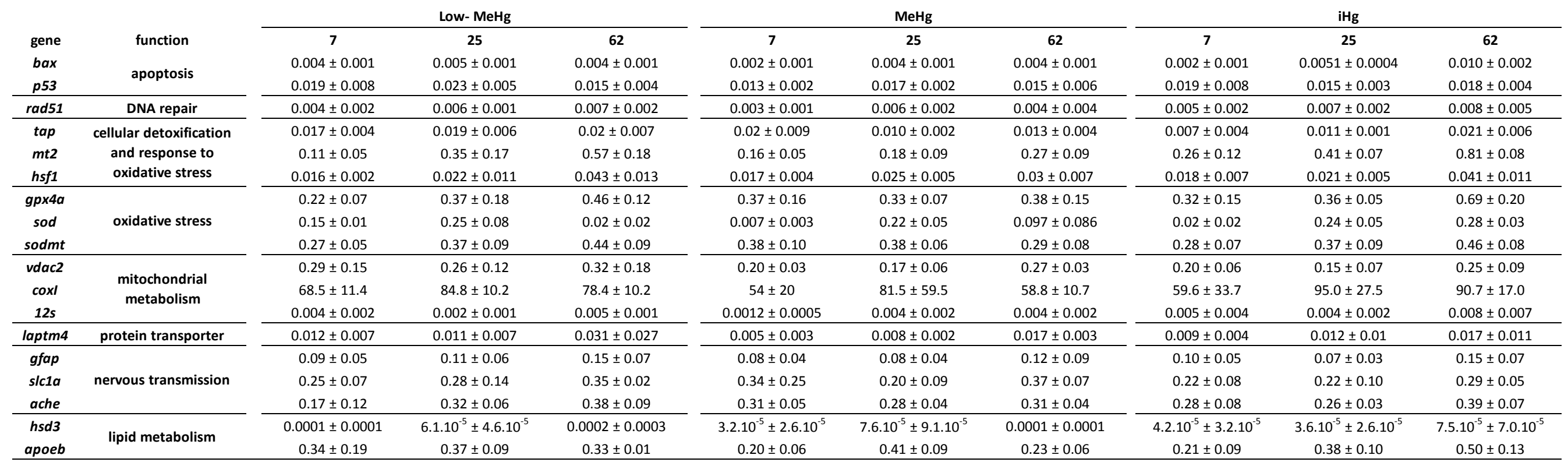


Figure 2S. Observation by electron microscopy of zebrafish skeletal muscle and liver exposed to dietary $\mathrm{MeHg}$ and iHg at $7 \mathrm{days}$. Legend muscle: M: mitochondria; F: muscle fiber, I: inter fiber bundle. Legend liver: H: Hepatocyte; N: nucleus; BC: Bile canaliculus; 1: lipids; L: lysosome/peroxisome; Ma: macrophage; Er: Endoplasmic reticulum.

\section{MUSCLE:}
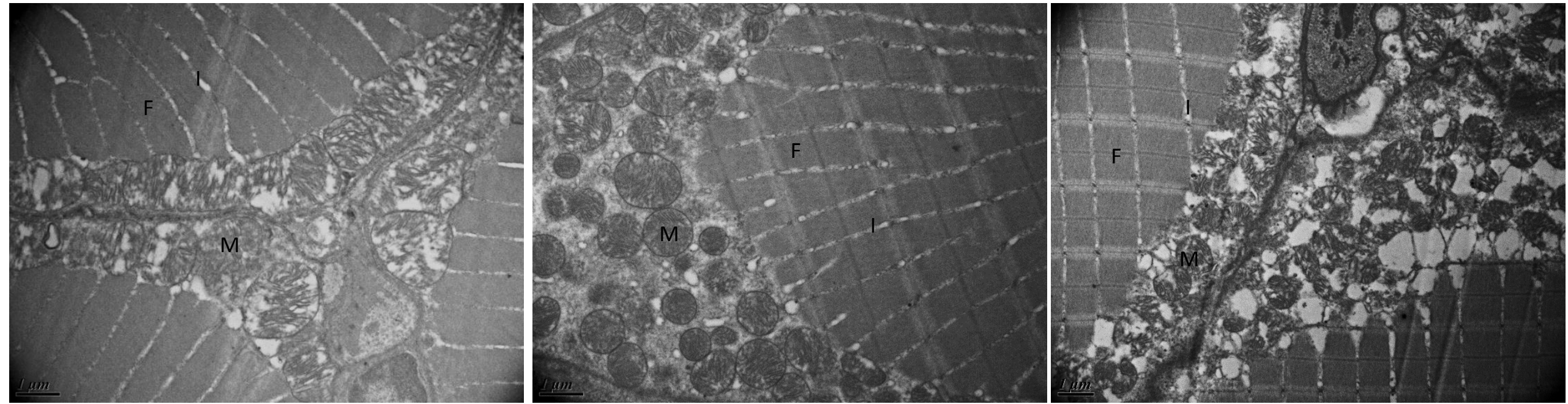

LIVER:
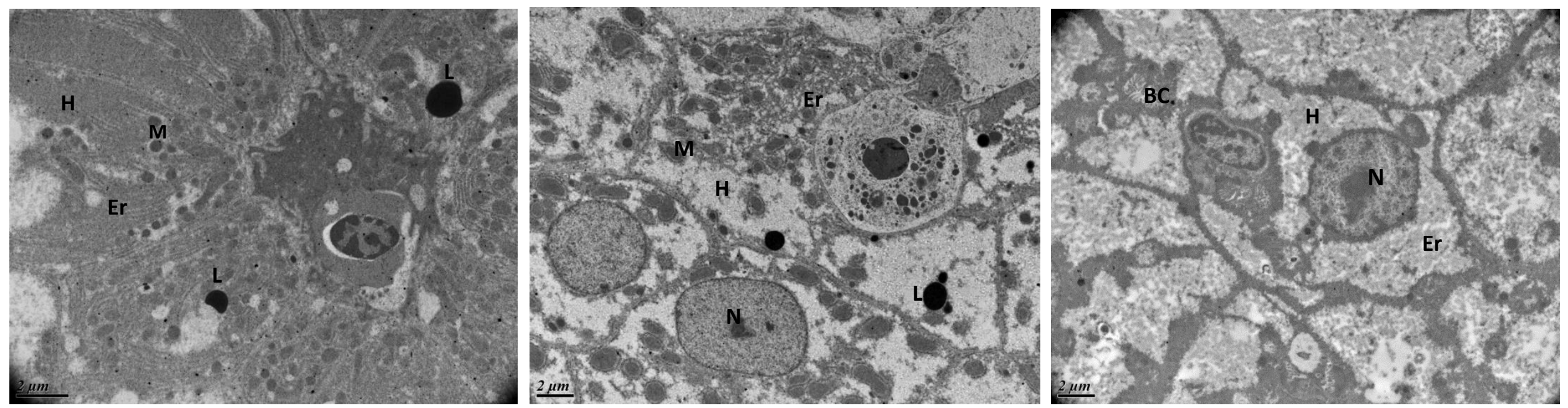